OU-HET 412

SU-ITP-02/20

UOSTP-02103

hep-th/0205265

\title{
Supersymmetric Brane-Antibrane Systems: Matrix Model Description, Stability and Decoupling Limits
}

\author{
Dongsu Bak ${ }^{a}$, Nobuyoshi Ohta ${ }^{b}$ and Mohammad M. Sheikh-Jabbari ${ }^{c}$ \\ ${ }^{a}$ Physics Department, University of Seoul, Seoul 130-743, Korea \\ ${ }^{b}$ Department of Physics, Osaka University, Toyonaka, Osaka 560-0043, Japan \\ ${ }^{c}$ Department of Physics, Stanford University, Stanford CA 94305-4060, USA \\ (dsbak@mach.uos.ac.kr, ohta@phys.sci.osaka-u.ac.jp, jabbari@itp.stanford.edu)
}

\begin{abstract}
After reviewing the supertubes and super brane-antibrane systems in the context of matrix model, we look for more general higher-dimensional configurations. For D3$\overline{\mathrm{D} 3}$, we find a non-trivial configuration with $\mathbf{E} \cdot \mathbf{B} \neq 0$ and describe the worldvolume gauge theory. We present the string probe of D3-D3 system and study the decoupling limits leading to either noncommutative Super-Yang-Mills or NCOS theories with eight supercharges.
\end{abstract}

November 3, 2018 


\section{Introduction}

After the stringy realization of D-branes [1], various brane configurations which preserve some supersymmetry and hence are stable, have been considered. Among these there are the interesting class of 1/4 BPS branes, D2-branes of tubular shape generally called supertubes [2]. The supertubes have also been realized in the matrix theory in Ref. [3], and it has been shown that it is possible to generalize the tubular configuration to $p$-branes whose worldvolumes are $R^{p-1} \times \mathcal{C}$, where $\mathcal{C}$ can be a circle or an ellipse (or even a hyperbola) [4]. Later it was proven that $\mathcal{C}$ can be a completely arbitrary curve [5].

There are simple but intriguing type of $1 / 4$ BPS brane systems, the supersymmetric brane anti-branes, which can be thought as a specific limit of the tubular branes. To see the relation, consider a supertube of elliptic cross section. In the limit that the longer axis of the ellipse goes to infinity while the shorter one is kept fixed, the supertube system becomes the flat brane-antibrane system [㭡. The spectrum and stability of the system were studied in Refs. [5, 6] and it was shown that the brane-antibrane tachyon is stabilized by proper amount of static electric and magnetic fields on the worldvolume of the branes. More precisely, for the case of D2- $\overline{\mathrm{D} 2}$ branes, the system is stabilized by distributing the proper amount of D0-branes and F-strings on the worldvolume of D2 and $\overline{\mathrm{D} 2}$. Other aspects of supertubes have been discussed in [0, 8, 9, 10, 11]. Related configurations of D2 supertubes ending on D4 have also been studied through the Born-Infeld action [12].

In this paper first we review the matrix model solutions of a $R \times \mathcal{C}$ type supertubes as well as $1 / 4$ BPS D2- $\overline{\mathrm{D} 2}$ system where, for the stability of the system, one needs a very specific electric field along the $R$ direction (axis of the supertube). However, the corresponding magnetic field can be chosen arbitrarily (provided that it is non-zero). Within the matrix theory, we illustrate how tubes with arbitrary cross sectional shape can be realized. By a slight modification of the 1/4 BPS equations, we also construct the solution of string-type spike pulled out of D2 with constant B-field background. Note that the "source term" present in this configuration is compatible with the supersymmetry condition. These solutions can be easily generalized to the cases of $p>2$.

In section 3, we focus on the D3- $\overline{\mathrm{D} 3}$ system and consider the worldvolume gauge field configurations with $\mathbf{E} \cdot \mathbf{B} \neq 0$. The supersymmetric D3- $\overline{\mathrm{D} 3}$ systems, having less supersymmetries (compared to individual branes) and of higher dimensions, can be of much interest from phenomenological point of view. We show that choosing the electric field of the proper value, i.e. $E=1$ in the conventions of this section, independently of the value of the magnetic field we can have a 1/4 BPS system. We also analyze this system from the Born-Infeld point of view. In section 4, starting from the matrix strings action [13], we present the matrix theory description of D3- $\overline{\mathrm{D} 3}$ system. We also present related BPS equations for less number of supercharges.

In section 5, we study the spectrum of open strings stretched between D3 and $\overline{\mathrm{D} 3}$ branes. We show that for a general background $B_{\mu \nu}$ field there is a tachyon in the spectrum. However, this tachyon becomes massless in the $E \rightarrow 1$ limit, compatible with our arguments of previous sections. In section 6, we study the NCSYM limit of the D3-D3 brane system. Performing the Seiberg-Witten limit [14] we show that such a decoupling limit, where the massless open strings are decoupled from the bulk supergravity modes, exists. In section 7 , we show that the D3- $\overline{\mathrm{D} 3}$ brane system admits another "decoupling limit", the noncommutative open string (NCOS) limit. In this limit, unlike 
the NCSYM decoupling limit of section 6, we have a non-critical open string theory, decoupled from all the closed string modes. We show that the existence of NCOS limit does not depend on the value of the background magnetic fields (provided that $\mathbf{E} \times \mathbf{B}$ is non-zero). This will provide a new class of NCOS theories with 8 supercharges. The last section is devoted to conclusions and discussions.

\section{Supertubes in the matrix model}

In this section, we first briefly review how the circular supertubes and super brane-antibrane systems arise from the matrix model [3, 7]. We then construct the tubes having an arbitrary cross section within the matrix model. We further construct the $1 / 4$ BPS configuration corresponding to (IIA) strings pulled out from noncommutative D2-branes. This configuration arises in close connection with the tubular geometry but requires a slight modification of the BPS equation. In fact this corresponds to the radius varying tubular configuration discussed in Ref. [2].

We begin with the matrix model Lagrangian [15]

$$
L=\frac{1}{2 R} \operatorname{tr}\left(\sum_{I}\left(D_{0} X_{I}\right)^{2}+\frac{1}{\left(2 \pi \alpha^{\prime}\right)^{2}} \sum_{I<J}\left[X_{I}, X_{J}\right]^{2}+\text { fermionic part }\right),
$$

where $I, J=1,2, \cdots 9, R=g_{s} l_{s}$ is the radius of the tenth spatial direction, and $\alpha^{\prime}\left(\equiv l_{s}^{2}\right)$ is related to the eleven-dimensional Planck length by $l_{11}=\left(R \alpha^{\prime}\right)^{\frac{1}{3}}$. The scale $R$ together with $2 \pi \alpha^{\prime}$ will be omitted below by setting them unity. We would like to emphasize that all our discussions are valid for any finite $R$ and $\alpha^{\prime}$ and one does not need any further decoupling limit for the validity of the description [16].

For the tubular configurations, we note that the supersymmetric variation of the fermionic coordinates $\psi$ in the matrix theory is

$$
\delta \psi=\left(D_{0} X^{I} \gamma_{I}+\frac{i}{2}\left[X^{I}, X^{J}\right] \gamma_{I J}\right) \epsilon+\epsilon^{\prime},
$$

where $\epsilon$ and $\epsilon^{\prime}$ are real spinors of 16 components parameterizing total 32 supersymmetries. Here we shall turn on only the first three components of $X^{I}$, which will be denoted by $X, Y$ and $Z$. Introducing the real projection operator $P_{ \pm}=\left(1 \pm \gamma_{z}\right) / 2$, one may rewrite the variation of the fermionic coordinate as

$$
\begin{aligned}
\delta \psi & =\left[\left(D_{0} X+i[Z, X]\right) \gamma_{x}+\left(D_{0} Y+i[Z, Y]\right) \gamma_{y}-D_{0} Z+i[X, Y] \gamma_{x y}\right] P_{-} \epsilon \\
& +\left[\left(D_{0} X-i[Z, X]\right) \gamma_{x}+\left(D_{0} Y-i[Z, Y]\right) \gamma_{y}+D_{0} Z+i[X, Y] \gamma_{x y}\right] P_{+} \epsilon+\epsilon^{\prime}=0
\end{aligned}
$$

Let us choose $P_{+} \epsilon=0$ (or $P_{-} \epsilon=0$ ) and $\epsilon^{\prime}=0$ as remaining supersymmetries. Any non-trivial solution will then preserve the $1 / 4$ of the original 32 supersymmetries. The coefficient of $P_{-} \epsilon$ should vanish for the remaining supersymmetries; this leads to the BPS equations

$$
D_{0} X+i[Z, X]=0, \quad D_{0} Y+i[Z, Y]=0, \quad D_{0} Z=0, \quad[X, Y]=0 .
$$


In addition one has to satisfy the Gauss law constraint,

$$
\left[X, D_{0} X\right]+\left[Y, D_{0} Y\right]+\left[Z, D_{0} Z\right]=0
$$

With the choice of the gauge $A_{0}=Z$, the BPS equations are reduced to

$$
[X,[X, Z]]+[Y,[Y, Z]]=0,[X, Y]=0,
$$

with time independent $X_{i}, i=1,2,3$. The circular tube solution is given by the representation of the algebra,

$$
[z, x]=i l y, \quad[y, z]=i l x, \quad[x, y]=0,
$$

with $X_{i}=x_{i}$. The shape is described by the Casimir $\rho^{2}=x^{2}+y^{2}$, which is proportional to identity for any irreducible representations of the algebra. The irreducible representations in the basis diagonalizing $Z$ are given as [3]

$$
x+i y=\rho \sum_{n=-\infty}^{\infty}|n+1\rangle\left\langle n\left|, \quad z=l \sum_{n=-\infty}^{\infty}(n+\alpha)\right| n\right\rangle\langle n|,
$$

with $\alpha \in[0,1)$. One can check that the configuration has a circular cross section at any fixed $z$. The location of D0-branes are equally spaced by $l$ in the $z$-direction having the lattice translational symmetry. One may generalize the solutions to those of $1 / 4$ BPS multi tubes [3]. This implies that there is no force between these tubes. The momentum density $D_{0} X_{i}$ is conserved and the central charge measures the F-string charges [4, 可].

The elliptic/hyperbolic deformation,

$$
[z, x]=i a y, \quad[y, z]=i b x, \quad[x, y]=0,
$$

of the algebra also solves the BPS equations for arbitrary constants $a$ and $b$. With the Casimir operators $\mathcal{K}=x^{2} / a+y^{2} / b$, the shape is elliptic/hyperbolic if $a b>0 / a b<0$. When $a b<0$, the configuration is in general composed of two separated sheets for a given $\mathcal{K}$; For $\mathcal{K}>0, a>0$ and $b<0$ the representation is

$$
x= \pm \sqrt{a \mathcal{K}} \cosh \kappa, \quad y=\sqrt{|b| \mathcal{K}} \sinh \kappa,
$$

with $[\kappa, z]=i \sqrt{|a b|}$. Each sheet of \pm signature is associated with an irreducible representation of the algebra.

If one takes the limit where $b(>0)$ goes to infinity while fixing $a(>0)$, the cross sectional shape becomes two parallel lines extended in $y$ direction. Since the total D2 charge of this system is zero, the configuration in this case corresponds to brane-antibrane system extended in $y$ - $z$ directions, which is $1 / 4$ BPS.

In fact the elliptic/hyperbolic deformation is not the most general case. The tubes of arbitrary cross sections are possible [5]. To see this, let us consider the configuration defined by

$$
X=F_{x}(x, y), \quad Y=F_{y}(x, y), \quad Z=z,
$$

\footnotetext{
${ }^{1}$ The time independence here does not mean that the momentum $D_{0} X_{i}$ should vanish.
} 
where $x, y$ and $z$ are the solutions of (2.9) and $F_{x}$ and $F_{y}$ are arbitrary real functions of $x$ and $y$. One may then easily see that this configuration solves the BPS equations (2.6). Since this amounts to an arbitrary coordinate transformation in $x-y$ plane, the curve defined by $x^{2}(X, Y) / a+$ $y^{2}(X, Y) / b=\mathcal{K}$ takes an arbitrary shape in $(X, Y)$ space. To obtain a curve of an arbitrary shape it is enough to introduce one arbitrary function, say, $F_{x}$. The remaining may be associated with the reparametrization of the coordinate describing the curve for a given shape. Because of this freedom, the worldvolume $B$-field become an arbitrary function along the curve but still independent of $z$. Due to the noncommutative description of the complicated shape, the argument here may look unclear. We illustrate this freedom explicitly for the case of flat brane solutions.

However before that we would like to note that there are closely related more general solutions:

$$
X=F_{x}(x, y), \quad Y=F_{y}(x, y), \quad Z=\frac{1}{2}(z H(x, y)+H(x, y) z)+G(x, y),
$$

where $H$ and $G$ are arbitrary real functions. This solution is related to 2.11) by a residual worldvolume reparametrization of the $Z$-coordinate. The BPS equations in (2.6) may be generalized

$$
\left[X_{k},\left[X_{k}, X_{9}\right]\right]=0, \quad\left[X_{k}, X_{l}\right]=0,
$$

with $k, l=1,2, \cdots 8$. Then the arbitrary curve may be generalized as

$$
X_{k}=F_{k}(x, y), \quad X_{9}=z,
$$

which describes an arbitrary curve in the eight-dimensional space transverse to $X_{9}$.

Now let us consider the planar solutions leading to super brane-antibrane systems. The configuration is again a particular solution of the above BPS equations

$$
[y, z]=i \Theta \otimes I, \quad x=A \otimes I,
$$

where $\Theta$ and $A$ are the diagonal $p \times p$ matrices. Here $p$ is the total number of branes and the signatures of the diagonal elements $\Theta_{n}$ represent whether the constituent corresponds to a brane or an antibrane. When $\Theta$ is proportional to identity, the configuration becomes $1 / 2$ BPS describing $p$ D2-branes in a constant NS-NS $B$-field background. This enhancement of supersymmetries may be directly checked using (2.3) with an appropriate $\epsilon^{\prime}$.

For the simple brane-antibrane system [4], one may take $p=2$ with $\Theta=\operatorname{diag}\left(\theta_{1},-\theta_{2}\right)$ and $A=$ $\operatorname{diag}\left(a_{1}, a_{2}\right)$ with $\theta_{1}, \theta_{2}>0$. The parameter $\theta$ describes the noncommutativity of the worldvolume theory and $a_{1}$ and $a_{2}$ describe the locations of the brane and antibrane in $x$-direction. Let us first consider $p=1$ case; the simplest solution is

$$
[y, z]=i \theta, \quad x=0
$$

This corresponds to the NS-NS background $B_{23}=-1 / \theta$. It is straightforward to check that the arbitrary $z$-independent $B$-field, described by

$$
Y=y+\theta h(y), \quad Z=z
$$


where $h$ is an arbitrary real function and $[y, z]=i \theta$, solves the BPS equations and the shape is still planar. However, if we define gauge field on the brane as

$$
Y=y+\theta A_{z}, \quad Z=z-\theta A_{y},
$$

then

$$
[Y, Z]=i\left(\theta+\theta^{2} F_{y z}\right)
$$

Therefore the solution (2.17) corresponds to the magnetic field $F_{y z}=h^{\prime}(y)$. The background NS-NS $B$-field responsible for the noncommutativity is $B_{23}=-1 / \theta$ with all the other components vanishing. Thus this planar configuration describes a planar brane with an arbitrary $B$-field. In this matrix formulation, the worldvolume dynamics is automatically described by a noncommutative field theory. The map to commutative variables is not that straightforward. They are, in fact, related by the Seiberg-Witten map [14];

$$
\begin{aligned}
& A_{\mu}=a_{\mu}-\frac{1}{4} \theta^{\alpha \beta}\left\{a_{\alpha}, \partial_{\beta} a_{\mu}+f_{\beta \mu}\right\}+O\left(\theta^{2}\right), \\
& F_{\mu \nu}=f_{\mu \nu}+\frac{1}{4} \theta^{\alpha \beta}\left(2\left\{f_{\mu \alpha}, f_{\nu \beta}\right\}-\left\{a_{\alpha}, D_{\beta} f_{\mu \nu}+\partial_{\beta} f_{\mu \nu}\right\}\right)+O\left(\theta^{2}\right),
\end{aligned}
$$

where $\{a, b\}=a b+b a$, the lower case letters are used for the commutative variables and the capital letter for the noncommutative ones. The electric field in this solution is evaluated as $F_{0 z} \equiv D_{z} A_{0}-\dot{A}_{z}=1+\theta h^{\prime}(y)$. Using the Seiberg-Witten map one may confirm that

$$
f_{0 y}=O\left(\theta^{2}\right), \quad f_{0 z}=1+O\left(\theta^{2}\right), \quad f_{y z}=h^{\prime}(y)-\frac{\theta}{2}\left(h^{2}(y)\right)^{\prime \prime}+O\left(\theta^{2}\right),
$$

while all the other components are vanishing. Hence we see here that, to the first order in $\theta$, the electric components agree with the commutative one.2 Also note that $f_{y z}$ or the combination with NS-NS background $-1 / \theta+f_{y z}$ involve an arbitrary function of $y$, which is in agreement with the commutative analysis [5]. The agreement is expected to hold to higher orders in $\theta$ but the confirmation requires the Seiberg-Witten map to higher orders.

Now we discuss another generalization of the BPS equations (2.4). Again we turn on the first three component of $X_{I}$. Using the projection $P_{+} \epsilon=0$ and taking $\epsilon^{\prime}=\theta \gamma_{12} \epsilon$, we obtain the following slightly generalized BPS equations:

$$
D_{0} X+i[Z, X]=0, \quad D_{0} Y+i[Z, Y]=0, \quad D_{0} Z=0, \quad[X, Y]=i \theta .
$$

With the Gauss law and the gauge choice $A_{0}=Z$, the BPS equations are reduced to

$$
[X,[X, Z]]+[Y,[Y, Z]]=0, \quad[X, Y]=i \theta .
$$

Without loss of generality, we can assume $\theta>0$. Introducing $a=(X+i Y) / \sqrt{2 \theta}$ and $a^{\dagger}=$ $(X-i Y) / \sqrt{2 \theta}$, the second equation implies $\left[a, a^{\dagger}\right]=1$. In the basis where the number operator $\hat{n}=a^{\dagger} a$ is diagonal, the solution reads

$$
X-i Y=x-i y=\sqrt{2 \theta} \sum_{n=0}^{\infty} \sqrt{n+1}|n+1\rangle\langle n| .
$$

\footnotetext{
${ }^{2}$ It should be noted that only the gauge invariant combination $(B+f)_{\mu \nu}$ has a physical meaning.
} 
Observe that the area preserving scaling $\bar{X}=e^{\omega} X$ and $\bar{Y}=e^{-\omega} Y$ for real $\omega$, generates a new solution. However, these solutions are related by the following unitary transformation: $\bar{x}_{i}=$ $e^{-\omega\left(a^{\dagger 2}-a^{2}\right)} x_{i} e^{\omega\left(a^{\dagger 2}-a^{2}\right)}$. It does not belong to the gauge transformation as, it does not satisfy the boundary condition at infinity. Note that the solution in $(2.23)$ is the one respecting the rotational symmetry. Using the solution (2.23), the first BPS equation in (2.22) becomes the Laplace equation

$$
\left(\partial_{x}^{2}+\partial_{y}^{2}\right) Z=0
$$

where we define $\left[x_{a}, \cdot\right]=i \theta \epsilon_{a b} \partial_{b}$ with $a, b=1,2$. In fact using the well known Moyal-Weyl map, $Z$ can be treated as an ordinary function in $x-y$ space. In terms of the ordinary function $\bar{Z}(x, y)$, Eq. (2.24) becomes the usual Laplace equation and the radially symmetric solution reads

$$
\bar{Z}=\frac{Q}{2 \pi} \ln \left(r / r_{0}\right)
$$

where we introduced "source", $Q \delta^{2}(x, y)$, at $z=-\infty$ (or $\left.r=0\right)$ to the Laplace equation. Note that the introduction of source term does not modify the BPS conditions in (2.21). The configuration still preserves $1 / 4$ of the supersymmetries everywhere. The introduction of source term merely modifies the Gauss law; the source may be consistently incorporated into the original Lagrangian (2.1) by a coupling to external charged sources.

This solution corresponds to a D2-brane with constant $B$-field pulled out by string. This is precisely the radius varying solution discussed in Ref. [2]. Noting $D_{0} X_{a}=-\theta \epsilon_{a b} E_{b}$, the electric field evaluated in terms of functions becomes $E_{a}=\frac{Q}{2 \pi} x_{a} / r^{2}$ and indeed one can confirm that the source has the electric charge $Q$. Furthermore if one uses $z, \varphi$ coordinates $(\varphi$ is defined by $\tan \varphi=y / x$ ) $F_{0 z}=\partial_{z} A_{0}$ and hence $E_{z}=1$, in agreement with Ref. [2]. The logarithmic behavior comes from the two-dimensional nature of D2-branes. The presence of point source at the origin makes the D2-brane get deformed even asymptotically as $r \rightarrow \infty$. This configuration may straightforwardly be generalized to higher dimensions in the T-dual setting of the above and then the branes become flat asymptotically.

The above solutions may be mapped to the operator representation [17] but we shall not delve into this detailed translation to the operator representation. Instead let us study the problem directly solving the matrix representations. Using the creation annihilation operator, the BPS equation plus the source becomes

$$
\frac{1}{\theta}\left(\left[a,\left[a^{\dagger}, Z\right]\right]+\left[a^{\dagger},[a, Z]\right]\right)=\hat{\rho},
$$

where $\hat{\rho}$ is the source. Although the precise nature of the source term is beyond the scope of the classical description, the natural choice from the view point of the noncommutative description would be

$$
\hat{\rho}=\frac{Q}{2 \pi \theta}|0\rangle\langle 0|
$$

which corresponds to the minimal unit of area at the origin. 3. (One may build up general sources superposing those obtained by arbitrary translation of the above source.) The solution reads

$$
Z=\sum_{n=0}^{\infty}\left(z_{0}+\frac{Q}{4 \pi} \sum_{k=1}^{n} \frac{1}{k}\right)|n\rangle\langle n| .
$$

\footnotetext{
3 This can be understood as a noncommutative point-like source, as discussed in 18 ].
} 
Noting that $r^{2}=2 \hat{n}+1$, the large $r$ behavior agrees with (2.25). For small $n$, the matrix solution (2.28) and the functional solution (2.25) do not agree because $|0\rangle\langle 0|$ corresponds not to a delta function source but a Gaussian type, in the function description.

\section{$3 \kappa$-symmetry of supersymmetric D3- $\overline{\mathrm{D} 3}$}

In the D2- $\overline{\mathrm{D} 2}$ system discussed in the previous section, the $B$-field components turned on are $B_{23}$ and $E_{z}=B_{30}$. As mentioned earlier, $E_{z}=1$ while $B_{23}$ may be an arbitrary function of $y$. The obvious higher-dimensional generalizations may be obtained by the T-duality actions along the transverse directions, e.g. in this way we can obtain a D3- $\overline{\mathrm{D} 3}$ system but with $E$ and $B$ fields satisfying $\mathbf{E} \cdot \mathbf{B}=0$.

Here we ask for more general configurations. In particular we consider turning on additional component of $B$ in the $z$-direction in D3- $\overline{\mathrm{D} 3}$ system. This will lead to the configuration of $\mathbf{E} \cdot \mathbf{B} \neq 0$. The supersymmetry of the configuration is determined by

$$
\Gamma \epsilon_{L}= \pm \epsilon_{R}
$$

where $\Gamma$ is the projection operator appearing in the worldvolume $\kappa$ symmetry and $+/-$ signs are respectively for brane/antibrane. The projection operator is determined as [19]

$$
\Gamma=\frac{\sqrt{g}}{\sqrt{g+B}}\left(\gamma_{0123}-\gamma_{12} E+\gamma_{03} \tilde{B}+\gamma_{01} B-E \tilde{B}\right),
$$

where $E=B_{03}, B=B_{23}$ and $\tilde{B}=B_{12}$. Then the condition (3.1) may be rearranged as

$$
\frac{\sqrt{g}}{\sqrt{g+B}}\left[\left(\gamma_{12}+\tilde{B}\right)\left(\gamma_{03}-E\right)+\gamma_{01} B\right] \epsilon_{L}= \pm \epsilon_{R} .
$$

When $E, B$ and $\tilde{B}$ vanish, the supersymmetry condition reduces to that of D3 (or $\overline{\mathrm{D} 3}$ )-branes, i.e. $\gamma_{0123} \epsilon_{L}= \pm \epsilon_{R}$. Without $B$-fields, it is clear that there is no remaining supersymmetry if both brane and antibrane are present. As in the case of supersymmetric D2- $\overline{\mathrm{D} 2}$ system, we set $E=1$ with projection $P_{+}^{z} \epsilon_{L}=\epsilon_{L}$ where we have defined

$$
P_{ \pm}^{z} \equiv\left(1 \pm \gamma_{03}\right) / 2
$$

Then the remaining supersymmetry condition becomest

$$
\operatorname{sign}(B) \gamma_{01} \epsilon_{L}= \pm \epsilon_{R} .
$$

This may be solved for both signs with the same $\epsilon_{R}$ if $B>0$ for + sign and $B<0$ for - sign. As in the case of supersymmetric D2- $\overline{\mathrm{D} 2}$, only the signature of $B$ is correlated to the brane charges and the magnitude is arbitrary. In addition $\tilde{B}$ is completely arbitrary and could change from brane to brane; the supersymmetries are not affected by them. The resulting configurations preserve $1 / 4$

\footnotetext{
${ }^{4}$ Note that here we have set $g_{\mu \nu}=\eta_{\mu \nu}$.
} 
of 32 supersymmetries. The supersymmetry condition by the above projection is that of stretched strings in $z$-direction and the condition in (3.5) agrees with supersymmetry condition for D-strings stretched in $x$-direction. This indicates that the system may be interpreted as a bound state of branes composed of F-strings and D-strings. The presence of $\tilde{B}$ implies that there are also D-strings stretched along $z$ direction inside the D3-branes.

In the above we have considered a constant $B$ and $E$ fields allowing variations from brane to brane. Let us now look more closely at the Born-Infeld description of such branes. The action is

$$
\mathcal{L}=-\sqrt{-\operatorname{det}(g+B)}=-\sqrt{1-\mathbf{E}^{2}+\mathbf{B}^{2}-(\mathbf{E} \cdot \mathbf{B})^{2}}=-\sqrt{\left(1-E^{2}\right)\left(1+\tilde{B}^{2}\right)+B^{2}},
$$

where we take $g_{\mu \nu}=\eta_{\mu \nu}$ for simplicity. However as we will see, it would be more convenient to consider more general closed string metric when we discuss the Seiberg-Witten decoupling, as well as the NCOS limit. The displacement becomes

$$
\boldsymbol{\Pi}=\frac{\partial \mathcal{L}}{\partial \mathbf{E}}=\frac{\mathbf{E}+\mathbf{B}(\mathbf{E} \cdot \mathbf{B})}{\sqrt{1-\mathbf{E}^{2}+\mathbf{B}^{2}-(\mathbf{E} \cdot \mathbf{B})^{2}}}
$$

and the Hamiltonian is given by

$$
\mathcal{H}=\sqrt{1+\mathbf{B}^{2}+\mathbf{\Pi}^{2}+(\mathbf{\Pi} \times \mathbf{B})^{2}}
$$

This may be arranged to the complete squared form as

$$
\mathcal{H}=\sqrt{\left(\sqrt{1+\tilde{B}^{2}}-\frac{\Pi|B|}{\sqrt{1+\tilde{B}^{2}}}\right)^{2}+\left(\tilde{\Pi} \sqrt{1+\tilde{B}^{2}}-\frac{\Pi B \tilde{B}}{\sqrt{1+\tilde{B}^{2}}}\right)^{2}+(|B|+\Pi)^{2}} \geq|B|+\Pi,
$$

where $\Pi=\Pi_{z}$ and $\tilde{\Pi}=\Pi_{x}$. This analysis leads to the BPS equations

$$
|B| \Pi=1+\tilde{B}^{2}, \quad \tilde{\Pi}\left(1+\tilde{B}^{2}\right)=\Pi B \tilde{B},
$$

which implies $E^{2}=1$.

The Bianchi identity $d F=0$ and the Gauss law constraint $\partial_{z} \Pi+\partial_{x} \tilde{\Pi}=0$ lead to the conditions

$$
\partial_{x} B+\partial_{z} \tilde{B}=0, \quad \partial_{z}\left(\frac{1+\tilde{B}^{2}}{|B|}\right)+\partial_{x}\left(\tilde{B} \frac{B}{|B|}\right)=0 .
$$

With these, one may check explicitly that all the equations of motion are satisfied.

Assuming $\partial_{z} B=\partial_{z} \tilde{B}=0$, the above conditions imply that $B$ and $\tilde{B}$ are arbitrary functions of $y$ variable only, i.e.

$$
B=B(y), \quad \tilde{B}=\tilde{B}(y), \quad E=1 .
$$

These $1 / 4$ BPS configurations have a translational symmetry in $z$ and $x$-direction.

We do not know how to solve (3.11) in general and any nontrivial solution depending on $x$ or $z$ would be quite interesting. A particular example of the solution with $\partial_{z} B \neq 0$ may be given by $B=C x \sec ^{2} C z$ and $\tilde{B}=-\tan C z$, which are singular at $C z=n \pi / 2$ with odd integer $n$. 


\section{Matrix model description of D3- $\overline{\mathrm{D} 3}$ system}

In this section, we will try to reconstruct the D3- $\overline{\mathrm{D} 3}$ using the matrix model. Specifically we shall use the matrix model compactified on a circle, matrix strings [13], by which one may obtain branes in IIB theory compactified on the dual circle. The bosonic part of the Lagrangian is 13]

$$
L=\frac{1}{2} \int d \sigma \operatorname{tr}\left(\left(\mathcal{F}_{t \sigma}\right)^{2}+\sum_{I}\left(D_{0} X_{I}\right)^{2}-\left(D_{\sigma} X_{I}\right)^{2}+\sum_{I<J}\left[X_{I}, X_{J}\right]^{2}+\text { fermionic part }\right),
$$

where $I, J=2, \cdots, 9$ and the matrix $X_{I}$ are function of $(t, \sigma)$. From the original matrix model, the $X^{1}$-direction is compactified on the circle and hence after the T-duality it is replaced with the gauge field $A_{\sigma}$ in the above Lagrangian and we consider the theory in the decompactification limit of the large dual circle.

The BPS equation related to the previous discussion may be obtained from the variation of the fermionic coordinates. Alternatively one may obtain the BPS equations by studying the Hamiltonian. Here we follow the latter method. Consider again turning on only $X, Y, Z$ of the transverse components. The Hamiltonian can be arranged as

$$
\begin{aligned}
H= & \frac{1}{2} \operatorname{tr}\left(\left(\mathcal{F}_{t \sigma}-D_{\sigma} Z\right)^{2}+\left(D_{0} X+i[Z, X]\right)^{2}+\left(D_{0} Y+i[Z, Y]\right)^{2}\right. \\
& \left.+\left(D_{0} Z\right)^{2}+\left(D_{\sigma} X\right)^{2}+\left(D_{\sigma} Y\right)^{2}+|[X, Y]|^{2}+2 C_{J}\right) \geq \operatorname{tr} C_{J}
\end{aligned}
$$

where the central charge $\operatorname{tr} C_{J}$ is defined by

$$
\operatorname{tr} C_{J}=i \operatorname{tr}\left(\left[X, Z\left(D_{0} X\right)\right]+\left[Y, Z\left(D_{0} Y\right)\right]+\partial_{\sigma}\left(\mathcal{F}_{t \sigma} Z\right)\right) .
$$

Note that to obtain (4.3) we have used the Gauss law constraint,

$$
-i\left[X_{I}, D_{0} X_{I}\right]+D_{\sigma} \mathcal{F}_{t \sigma}=0 .
$$

Then the BPS equations are

$$
\begin{aligned}
& \mathcal{F}_{t \sigma}-D_{\sigma} Z=0, \quad D_{0} X+i[Z, X]=0, \quad D_{0} Y+i[Z, Y]=0 \\
& {[X, Y]=0, \quad D_{0} Z=D_{\sigma} X=D_{\sigma} Y=0}
\end{aligned}
$$

Choosing the gauge $A_{0}=Z$, the BPS equations imply that all the matrix variables should be static. Together with the Gauss law (4.4), the BPS equations (4.5) are reduced to

$$
\begin{aligned}
& {[X,[X, Z]]+[Y,[Y, Z]]+D_{\sigma}^{2} Z=0} \\
& {[X, Y]=0, \quad D_{\sigma} X=D_{\sigma} Y=0 .}
\end{aligned}
$$

To obtain the BPS equations one could use the remaining supersymmetry condition using the variation of the fermionic coordinate. The $1 / 4 \mathrm{BPS}$ condition we are interested in is the T-dual version of the tubular branes. Note that there we have used the projection $P_{ \pm}=\left(1 \pm \gamma_{z}\right) / 2$. While keeping this projection, the condition $\mathcal{F}_{t \sigma}=D_{\sigma} Z$ is inevitable for the $1 / 4$ BPS configuration. 
Before solving these BPS equations, we would like to record here more general form of the BPS equations by turning on the remaining transverse coordinates. By a similar method, it is straightforward to show that the resulting BPS equations are

$$
\begin{aligned}
& \sum_{k=2}^{8}\left[X_{k},\left[X_{k}, X_{9}\right]\right]+D_{\sigma}^{2} X_{9}=0 \\
& {\left[X_{k}, X_{l}\right]=0, \quad D_{\sigma} X_{k}=0}
\end{aligned}
$$

where $k, l=2,3, \cdots 8$.

The T-dual version of the above BPS equations are already given in (2.13). Further generalization analogous to $(2.22)$ is given by

$$
\sum_{k=1}^{8}\left[X_{k},\left[X_{k}, X_{9}\right]\right]=0, \quad\left[X_{k}, X_{l}\right]=i \theta_{k l},
$$

where $k, l=1,2 \cdots 8$. There are solutions of this BPS equation generalizing the IIA D2p-branes in a constant NS-NS $B$ field background pulled out by fundamental strings. We shall not repeat here the previous discussion. Similar configuration can be found in the IIB theory generalizing (4.7).

The above sets of BPS equations are direct generalizations of (2.6) and (2.22) to higher dimensions. Their solutions preserve at least a quarter of the original 32 supersymmetries. There are other generalizations involving smaller fractions. For example, the BPS equations for $1 / 8$ supersymmetries may be constructed as follows. We first introduce projection operators

$$
P_{ \pm}^{9}=\frac{1 \pm \gamma_{9}}{2}, \quad P_{ \pm}^{1234}=\frac{1 \pm \gamma_{1234}}{2}
$$

which are real and commute with each other. Using the variation of the fermionic coordinate in (2.2), we consider the cases with

$$
\begin{aligned}
P_{-}^{9} \epsilon & =0, \quad P_{-}^{1234} \epsilon=0 \\
\epsilon^{\prime} & =\frac{1}{2} \theta^{m n} \gamma_{m n} \epsilon, \quad m, n, p, q=1,2,3,4
\end{aligned}
$$

This leads to the BPS equations

$$
D_{0} X^{m}=-i\left[X^{9}, X^{m}\right], \quad D_{0} X^{9}=0, \quad\left[X^{m}, X^{n}\right]-i \theta^{m n}=\frac{1}{2} \epsilon_{m n p q}\left(\left[X^{p}, X^{q}\right]-i \theta^{p q}\right)
$$

with the Gauss constraint

$$
\left[X^{m}, D_{0} X^{m}\right]=0 .
$$

In the gauge where $A_{0}=X^{9}$, these equations are reduced to

$$
\begin{aligned}
& {\left[X^{m}, X^{n}\right]-i \theta^{m n}=\frac{1}{2} \epsilon_{m n p q}\left(\left[X^{p}, X^{q}\right]-i \theta^{p q}\right)} \\
& {\left[X^{m},\left[X^{m}, X^{9}\right]\right]=0,}
\end{aligned}
$$

with all the variables independent of time. The first equation here is for noncommutative instantons of D0-D4 systems and the second makes them dyonic. These are 1/8 BPS equations for the 
noncommutative version of the dyonic instantons [20] and the supertubes ending on D4-brane [12]. Detailed investigations for $U(1)$ as well as $U(p)$ would be quite interesting but we shall not attempt here to do so.

Now we get back to the BPS equations in (4.6). The solutions

$$
X=0, \quad Y=y+\theta h(y), \quad Z=z, \quad A_{\sigma}=0,
$$

with $[y, z]=i \theta$ correspond to the T-dual version of the flat D2 solution in (2.17). We shall identify the worldvolume directions as $x^{1}=\sigma, x^{2}=y$ and $x^{3}=z . X$ is then describing the transverse fluctuation of the D3-brane. Again using the definition

$$
Y=x^{2}+\theta A_{3}, \quad Z=x^{3}-\theta A_{2},
$$

one finds that

$$
[Y, Z]=i \theta+i \theta^{2} F_{23}, \quad D_{a} Z=\theta F_{a 2}, \quad D_{a} Y=-\theta F_{a 3}, \quad F_{t \sigma}=F_{01},
$$

with $a=0,1$. The solution (4.14) is a special case of above with $F_{12}=0$ and $F_{23}=h^{\prime}(y)$ and only the $B_{23}$ component of NS-NS two-form background is non-vanishing. Moreover, using the Seiberg-Witten map, one can show that $f_{03}=1$ and $f_{23}$ is an arbitrary function of $y$. Hence it is clear that the above solution is the T-dual version of (2.17) in the $\sigma$ direction. $\tilde{B}$

Let us now consider the case with non-zero $F_{12}$. There is a simple solution with non-vanishing

$$
X=0, \quad Y=y+\theta h(y), \quad Z=z, \quad A_{\sigma}=k(y),
$$

with $[y, z]=i \theta$. The solution stands for a flat brane and $h(y)$ and $k(y)$ are completely arbitrary functions of $y$. Of course there are more general solutions which depend on $x^{1}=\sigma$ but we shall restrict our consideration to the $\sigma$ independent cases. For the solution (4.17), $F_{y z}=h^{\prime}(y)$, $F_{12}=k^{\prime}(y)$ and $F_{t \sigma}=-\theta k^{\prime}$. At first sight, the comparison to that of the Born-Infeld analysis may seem problematic. Namely there is non-vanishing $F_{01}$ component (related to $F_{12}$ ), which was not present in the analysis of the previous section. However again the Seiberg-Witten map plays a non-trivial role. For the comparison, we again use the Seiberg-Witten map, Eqs. (2.19) to get the commutative variables. They are evaluated as

$$
\begin{aligned}
& f_{01}=f_{02}=f_{13}=O\left(\theta^{2}\right), \quad f_{03}=1+O\left(\theta^{2}\right), \\
& f_{12}=k^{\prime}(y)-\theta\left(h k^{\prime}\right)^{\prime}+O\left(\theta^{2}\right), \quad f_{23}=h^{\prime}(y)-\frac{\theta}{2}\left(h^{2}(y)\right)^{\prime \prime}+O\left(\theta^{2}\right) .
\end{aligned}
$$

Hence these are in agreement with the analysis of the previous section.

Many brane solutions generalizing (4.17) can be easily obtained in a similar way described in the previous section. Here we shall not exhaust all possible solutions of the above BPS equations. There are many obvious solutions. There exist solutions corresponding to the three-dimensional generalization of tubes of arbitrary cross section obtained by T-duality.

Finally let us consider the worldvolume dynamics around the supersymmetric background,

$$
X=0, \quad Y=y, \quad Z=z, \quad A_{\sigma}=\tilde{B} y
$$


with constant $\tilde{B}$ and $[y, z]=i \theta$. This corresponds to $h=0$ and $k=\tilde{B} y$ of the solution (4.17). The non-vanishing components of NS-NS two-form background is then $B_{23}=-1 / \theta, F_{12}=\tilde{B}$ and $F_{01}=-\theta \tilde{B}$. Through the computation of the open string metric on the worldvolume, we demonstrate that this solution precisely describes the supersymmetric background in (3.12) with constant $B=-1 / \theta$ and $\tilde{B}$. This identification is also consistent with the results in 4.18). To describe the worldvolume dynamics, let us define the worldvolume gauge fields $\mathcal{A}_{\mu}$ by

$$
\begin{array}{ll}
A_{0}=Z+\mathcal{A}_{0}, & A_{\sigma}=\tilde{B} Y+\mathcal{A}_{1} \\
Y=y+\theta \mathcal{A}_{3}, & Z=z-\theta \mathcal{A}_{2} .
\end{array}
$$

Also $X_{s}=\varphi_{s}$, where $X_{s}$ are other than $Y, Z$, become the six transverse scalar fields. Inserting these to the matrix model Lagrangian (4.1), one finds

$$
\begin{aligned}
L & =\frac{1}{2} \int d \sigma \operatorname{tr}\left[\mathcal{F}_{01}^{2}+\theta^{2} \mathcal{F}_{02}^{2}+\theta^{2}\left(1+\tilde{B}^{2}\right) \mathcal{F}_{03}^{2}-\theta^{2} \mathcal{F}_{13}^{2}+2 \theta\left(\mathcal{F}_{01}+\theta \tilde{B} \mathcal{F}_{03}\right)\left(\mathcal{F}_{12}+\theta \tilde{B} \mathcal{F}_{32}\right)\right. \\
& \left.+2 \theta \mathcal{F}_{03}\left(\tilde{B} \mathcal{F}_{01}-\theta \mathcal{F}_{23}\right)+\left(\nabla_{0} \varphi_{s}-\theta \nabla_{2} \varphi_{s}\right)^{2}-\left(\nabla_{1} \varphi_{s}+\theta \tilde{B} \nabla_{3} \varphi_{s}\right)^{2}-\theta^{2}\left(\nabla_{2} \varphi_{s}\right)^{2}-\theta^{2}\left(\nabla_{3} \varphi_{s}\right)^{2}\right]
\end{aligned}
$$

where

$$
\nabla_{\mu}=\partial_{\mu}-i\left[\mathcal{A}_{\mu},\right], \quad \mathcal{F}_{\mu \nu}=\partial_{\mu} \mathcal{A}_{\nu}-\partial_{\nu} \mathcal{A}_{\mu}-i\left[\mathcal{A}_{\mu}, \mathcal{A}_{\nu}\right]
$$

This Lagrangian may be written in a standard form

$$
L=-\frac{1}{4 g_{\mathrm{YM}}^{2}} \int d \sigma 2 \pi \theta \operatorname{tr} \sqrt{G}\left(G^{\alpha \mu} G^{\beta \nu} \mathcal{F}_{\alpha \beta} \mathcal{F}_{\mu \nu}+2 G^{\mu \nu} \nabla_{\mu} \varphi_{s} \nabla_{\nu} \varphi_{s}\right)
$$

with help of an appropriate metric $G^{\mu \nu}$. In section 6 , we shall explicitly show that the metric appearing here is precisely the open string metric associated with the background (3.12).

\section{$5 \quad$ D3- $\overline{\mathrm{D} 3}$ open string spectrum and stability}

As we have shown explicitly in section 3, we can have a $\frac{1}{4}$ BPS D3-D3 brane system with $B$-fields turned on them. In particular we discussed that there is a $B$-field configuration with $\mathbf{E} \cdot \mathbf{B} \neq 0$. These solutions are described by non-zero $B_{03}, B_{12}, B_{23}$ fields. Such D3- and $\overline{\mathrm{D} 3}$-branes can be obtained by T-duality in a tilted direction on the solutions of Ref. [4, 6], therefore we expect that there should not be a tachyonic mode in the open string spectrum stretched between the D3- and $\overline{\mathrm{D}}$-branes. For the later use and as an explicit check, in this section we work out the details of these open string spectrum.

The background fields at $\sigma=0$ (on the D3-brane) are

$$
B^{(0)}=\frac{1}{2 \pi \alpha^{\prime}}\left(\begin{array}{cccc}
0 & 0 & 0 & E \\
0 & 0 & \tilde{B} & 0 \\
0 & -\tilde{B} & 0 & B \\
-E & 0 & -B & 0
\end{array}\right) ; \quad g_{i j}=\eta_{i j}
$$


and as we have discussed earlier, at $\sigma=\pi$ (on the $\overline{\mathrm{D} 3}$-brane) $B^{(\pi)}$ is obtained by reversing the sign of $B$ and $\tilde{B}$. The boundary conditions are given as

$$
g_{i j} \partial_{\sigma} X^{j}+2 \pi \alpha^{\prime} B_{i j} \partial_{t} X^{j}=0, \quad \sigma=0, \pi .
$$

In a more explicit form they are

$$
\begin{aligned}
\partial_{\sigma} X^{0}-E \partial_{t} X^{3} & =0, \\
\partial_{\sigma} X^{1} \pm \tilde{B} \partial_{t} X^{2} & =0, \quad \text { at } \sigma=0, \pi, \\
\partial_{\sigma} X^{2} \mp \tilde{B} \partial_{t} X^{1} \pm B \partial_{t} X^{3} & =0, \\
\partial_{\sigma} X^{3}-E \partial_{t} X^{0} \mp B \partial_{t} X^{2} & =0 .
\end{aligned}
$$

The mode expansions are found to be

$$
\begin{aligned}
X^{0}= & \frac{1}{\sqrt{1-E^{2}}}\left(x^{0}+2 \alpha^{\prime} p_{0} t\right)+\frac{E \tilde{B}}{B} 2 \alpha^{\prime} p_{1} \sigma \\
& +i \sqrt{\frac{2 \alpha^{\prime}}{1-E^{2}}} \sum_{n \neq 0}\left[\sqrt{1+\frac{\tilde{B}^{2} E^{2}}{\rho^{2}}} \frac{a_{n}^{0}}{n} e^{-i n t} \cos n \sigma-\frac{E \tilde{B}}{\rho} \frac{a_{n}^{1}}{n} e^{-i n(t-\sigma)}\right] \\
- & i \frac{E B \sqrt{\alpha^{\prime}}}{\left(1-E^{2}\right) \rho} \sum\left(\frac{c_{n+\nu}}{n+\nu} e^{-i(n+\nu) t-i \frac{\pi \nu}{2}}-\frac{d_{-n-\nu}}{n+\nu} e^{i(n+\nu) t+i \frac{\pi \nu}{2}}\right) \sin \left[(n+\nu) \sigma-\frac{\pi \nu}{2}\right], \\
X^{1}= & x^{1}+2 \alpha^{\prime} p_{1} t+i \sqrt{\frac{2 \alpha^{\prime}}{1-E^{2}} \frac{B}{\rho} \sum_{n \neq 0} \frac{a_{n}^{1}}{n} e^{-i n t} \cos n \sigma} \\
& -\frac{\tilde{B} \sqrt{\alpha^{\prime}}}{\rho} \sum\left(\frac{c_{n+\nu}}{n+\nu} e^{-i(n+\nu) t-i \frac{\pi \nu}{2}}+\frac{d_{-n-\nu}}{n+\nu} e^{i(n+\nu) t+i \frac{\pi \nu}{2}}\right) \cos \left[(n+\nu) \sigma-\frac{\pi \nu}{2}\right], \\
X^{2}= & x^{2}+i \sqrt{\alpha^{\prime}} \sum\left(\frac{c_{n+\nu}}{n+\nu} e^{-i(n+\nu) t-i \frac{\pi \nu}{2}}-\frac{d_{-n-\nu}}{n+\nu} e^{i(n+\nu) t+i \frac{\pi \nu}{2}}\right) \cos \left[(n+\nu) \sigma-\frac{\pi \nu}{2}\right], \\
X^{3}= & x^{3}+\frac{\tilde{B}}{B} 2 \alpha^{\prime} p_{1} t+\frac{E}{\sqrt{1-E^{2}}} 2 \alpha^{\prime} p_{0} \sigma \\
& +\sqrt{\frac{2 \alpha^{\prime}}{1-E^{2}}} \sum_{n \neq 0}\left[E \sqrt{1+\frac{\tilde{B}^{2} E^{2}}{\rho^{2}}} \frac{a_{n}^{0}}{n} \sin n \sigma+i \frac{\tilde{B}}{\rho}\left(\cos n \sigma+i E^{2} \sin n \sigma\right) \frac{a_{n}^{1}}{n}\right] e^{-i n t} \\
& +\frac{B \sqrt{\alpha^{\prime}}}{\left(1-E^{2}\right) \rho} \sum\left(\frac{c_{n+\nu}}{n+\nu} e^{-i(n+\nu) t-i \frac{\pi \nu}{2}}+\frac{d_{-n-\nu}}{n+\nu} e^{i(n+\nu) t+i \frac{\pi \nu}{2}}\right) \cos \left[(n+\nu) \sigma-\frac{\pi \nu}{2}\right],
\end{aligned}
$$

where $\nu$ and $\rho$ are defined by

$$
\tan \frac{\pi \nu}{2}=\sqrt{\tilde{B}^{2}+\frac{B^{2}}{1-E^{2}}} \equiv \rho, \quad(0 \leq \nu<1),
$$

and the commutation relations of the mode operators are

$$
\left[c_{n+\nu}, d_{m-\nu}\right]=(n+\nu) \delta_{n+m}, \quad\left[a_{n}^{0}, a_{m}^{0}\right]=-n \delta_{n+m}, \quad\left[a_{n}^{1}, a_{m}^{1}\right]=n \delta_{n+m}, \quad\left[x^{\mu}, p_{\nu}\right]=i \eta^{\mu}{ }_{\nu} .
$$

This result shows that $\nu=1$ for $E=1$, in which case the levels are integer, which is in accordance with the supersymmetry restoration in the limit $E=1$.

${ }^{5}$ Though the mode expansions (5.4) look singular in the limit $E \rightarrow 1$, the worldsheet CFT is well defined just as for D2- $\overline{\mathrm{D} 2}$ system $[6]$. 
We define vacuum by $c_{n+\nu}|0\rangle=0$ for $n \geq 0$ and $d_{n-\nu}|0\rangle=0$ for $n>0$. Hence the corresponding vacuum energy from $X^{0}, X^{1}, X^{2}$ and $X^{3}$ becomes

$$
E_{\nu}=-\frac{1}{2} \sum_{n=1}^{\infty} n+\frac{1}{2} \sum_{n=1}^{\infty} n+\frac{1}{2} \sum_{n=1}^{\infty}(n-\nu)+\frac{1}{2} \sum_{n=1}^{\infty}(n-(1-\nu))=\frac{1}{24}-\frac{1}{8}(2 \nu-1)^{2},
$$

where we have used

$$
\mathcal{E}(a) \equiv \sum_{n=1}^{\infty}(n-a)=\frac{1}{24}-\frac{1}{8}(2 a-1)^{2}
$$

The vacuum energy for the Ramond sector is trivial; the bosonic one is canceled by the fermionic contribution and the total is zero. In the Neveu-Schwarz sector, the vacuum energy from the NSfermions associated with $0,1,2,3$ components is given by the substitution $\nu \rightarrow|\nu-1 / 2|$. In the "light-cone" gauge, the contributions from $a_{n}^{0}$ and one transverse oscillator cancel with each other. Summing the contributions from the rest of oscillators, we find the ground state energy

$$
\begin{aligned}
E_{\nu}^{\text {total }} & =\left(\frac{1}{24}-\frac{1}{8}(2 \nu-1)^{2}\right)-\left(\frac{1}{24}-\frac{1}{8}(2|\nu-1 / 2|-1)^{2}\right)-\frac{6}{24}-\frac{6}{48} \\
& =-\frac{1}{4}-\frac{|\nu-1 / 2|}{2} .
\end{aligned}
$$

For $\nu=0$, this is consistent with the vacuum energy of NS sector when the background $B$-fields are turned off.

The ground state energy (5.9) gives two states with energy

$$
\begin{array}{rlr}
E_{1}=-\frac{1}{2} \nu, & \text { for } & 1 \geq \nu \geq \frac{1}{2}, \\
E_{0}=\frac{1}{2}(\nu-1), & \text { for } & \frac{1}{2} \geq \nu \geq 0 .
\end{array}
$$

When $\nu=0,\left|E_{0}\right\rangle$ gives the true ground state and $\left|E_{1}\right\rangle$ is the first excited state, but the energy changes when $\nu$ is increased. For $\nu \geq \frac{1}{2},\left|E_{1}\right\rangle$ becomes the true ground state and $\left|E_{0}\right\rangle$ is the first excited state. For $\nu=0$ and D3-D3, the ground state $\left|E_{0}\right\rangle$ is projected out by GSO projection and $\left|E_{1}\right\rangle$ is kept. However, our D3- $\overline{\mathrm{D} 3}$ system has opposite GSO projection, and the state with $E_{1}$ is projected out and $\left|E_{0}\right\rangle$ is kept, leading to a tachyonic state. This state becomes massless for $\nu=1$ and hence for $E=1$ the system is tachyon free and stable.

Let us consider what is the spectrum at lower levels. The ground state is denoted by

$$
\left|-\frac{1}{2} \nu\right\rangle
$$

Corresponding to the mode oscillators $c_{n+\nu}$ and $d_{-n-\nu}$, we have fermionic oscillators $\psi_{n+\nu-\frac{1}{2}}$ and $\bar{\psi}_{-n-\nu-\frac{1}{2}}$. For $\nu \geq \frac{1}{2}, \bar{\psi}_{-\nu+\frac{1}{2}}, \psi_{\nu-\frac{3}{2}}$ and transverse oscillators $\psi_{-\frac{1}{2}}^{i}(i=3, \ldots, 8)$ give lower states (which remain after GSO projection)

$$
\left|\frac{1}{2}(\nu-1)\right\rangle \equiv \bar{\psi}_{-\nu+\frac{1}{2}}\left|-\frac{1}{2} \nu\right\rangle,
$$




$$
\begin{aligned}
\left|\frac{3}{2}(1-\nu)\right\rangle & \equiv \psi_{\nu-\frac{3}{2}}\left|-\frac{1}{2} \nu\right\rangle, \\
\left|\frac{1}{2}(1-\nu)\right\rangle^{i} & \equiv \psi_{-\frac{1}{2}}^{i}\left|-\frac{1}{2} \nu\right\rangle .
\end{aligned}
$$

For $\nu<1$, the first state is the ground state discussed above and gives tachyonic one. All these states give 8 massless for $\nu=1$, and the level is degenerate with Ramond sector, in accordance with the restoration of supersymmetry. Our discussions are quite analogous to those in Ref. [6]. The system has $1 / 4$ supersymmetry in the limit $E \rightarrow 1$. Our result of 8 massless bosonic states is consistent with this claim because the representation of 8 supercharges contains $2^{4}$ states in total, half of which are bosonic.

\section{NCSYM limit of D3- $\overline{\mathrm{D} 3}$ branes with $\mathbf{E} \cdot \mathbf{B} \neq 0$}

Here we would like to compute the metric for the noncommutative worldvolume theory on the D3-brane. For this, we shall follow the procedure described in Refs. [目, 21]. We begin with the closed string metric of the diagonal form $g_{\mu \nu}=\operatorname{diag}\left(-\left|g_{t}\right|, g_{x}, g_{y}, g_{z}\right)$ and $B_{\mu \nu}$ of the form given in (5.1), which is nothing but $B_{\mu \nu}$ for the D3-brane. With this metric and after restoring $2 \pi \alpha^{\prime}$ factors, the condition for supersymmetry or the "criticality" becomes

$$
\left|g_{t}\right| g_{y} g_{z}=g_{y}\left(2 \pi \alpha^{\prime} E\right)^{2}
$$

and hence, $\lambda E= \pm \sqrt{\left|g_{t}\right| g_{z}}$ with $\lambda=2 \pi \alpha^{\prime}$. The open string metric can be identified using the following relation to the closed string metric [21]:

$$
\frac{1}{g+\lambda B}=\frac{\theta}{\lambda}+\frac{1}{G+\lambda \Phi}
$$

where $G$ and $\theta^{\mu \nu}$ are the open string metric and the noncommutativity of the worldvolume theory, respectively. The two form $\Phi$ is free to choose but there is a natural one for the matrix theory description [21]; in the matrix theory they are given by the relation $\left[\hat{\partial}_{i}, \hat{\partial}_{j}\right]=-i \Phi_{i j}$ where $\hat{\partial}_{i}=$ $-i B_{i j} x^{j}$ and $\left[x^{i}, x^{j}\right]=i \theta^{i j}=i\left(B^{-1}\right)^{i j}$.

With the value $E$ in (6.1),

$$
\frac{1}{g+\lambda B}=-\frac{1}{\left|g_{t}\right| g_{x}(\lambda B)^{2}}\left(\begin{array}{cccc}
g_{x}(\lambda B)^{2}+g_{z} K & -\lambda B \lambda \tilde{B} \lambda E & -g_{x} \lambda E \lambda B & -\lambda E K \\
\lambda B \lambda \tilde{B} \lambda E & -\left|g_{t}\right|(\lambda B)^{2} & 0 & -\left|g_{t}\right| \lambda B \lambda \tilde{B} \\
-g_{x} \lambda E \lambda B & 0 & 0 & \left|g_{t}\right| g_{x} \lambda B \\
\lambda E K & -\left|g_{t}\right| \lambda B \lambda \tilde{B} & -\left|g_{t}\right| g_{x} \lambda B & -\left|g_{t}\right| K
\end{array}\right)
$$

where $K=g_{x} g_{y}+(\lambda \tilde{B})^{2}$. Then the Seiberg-Witten limit, resulting in a noncommutative gauge theory on branes, corresponds to

$$
\lambda \sim \sqrt{\epsilon}, \quad g_{y} \sim g_{z} \sim \epsilon, \quad \epsilon \rightarrow 0,
$$


keeping $B, \tilde{B}, g_{t}$ and $g_{x}$ fixed. From (6.1) it is seen that in this limit $E$ is also fixed. In this limit, one finds that

$$
\theta=\left(\begin{array}{cccc}
0 & 0 & 0 & 0 \\
0 & 0 & 0 & 0 \\
0 & 0 & 0 & -1 / B \\
0 & 0 & 1 / B & 0
\end{array}\right)
$$

for any fixed $\Phi$; this agrees with $\theta$ appearing in the matrix model description. The two form for the matrix model is identified as

$$
\Phi=\left(\begin{array}{cccc}
0 & 0 & 0 & 0 \\
0 & 0 & 0 & 0 \\
0 & 0 & 0 & -B \\
0 & 0 & B & 0
\end{array}\right)
$$

From this and using (6.2), one may compute $G_{\mu \nu}$ without taking the Seiberg-Witten limit:

$$
G_{\mu \nu}=\left((g+\lambda B)^{-1}-\frac{\theta}{\lambda}\right)_{\mu \nu}^{-1}-\lambda \Phi_{\mu \nu} .
$$

A straightforward evaluation yields

$$
G_{\mu \nu}=\left(\begin{array}{cccc}
0 & 0 & \lambda B \lambda E / g_{z} & 0 \\
0 & K / g_{y} & 0 & -\lambda B \lambda \tilde{B} / g_{y} \\
\lambda B \lambda E / g_{z} & 0 & (\lambda B)^{2} / g_{z} & 0 \\
0 & -\lambda B \lambda \tilde{B} / g_{y} & 0 & (\lambda B)^{2} / g_{y}
\end{array}\right),
$$

and

$$
G^{\mu \nu}=\left(\begin{array}{cccc}
-\frac{1}{\left|g_{t}\right|} & 0 & \frac{g_{z}}{\lambda E \lambda B} & 0 \\
0 & \frac{1}{g_{x}} & 0 & \frac{\lambda \tilde{B}}{g_{x} \lambda B} \\
\frac{g_{z}}{\lambda E \lambda B} & 0 & 0 & 0 \\
0 & \frac{\lambda \tilde{B}}{g_{x} \lambda B} & 0 & \frac{g_{x} g_{y}+(\lambda \tilde{B})^{2}}{g_{x}(\lambda B)^{2}}
\end{array}\right)
$$

We note that consistently (using coordinate transformations) we can set $\left|g_{t}\right|=g_{x}=1$, and also $\frac{g_{y}}{\lambda^{2}}=B^{2}-\tilde{B}^{2}$, then $\frac{g_{z}}{\lambda^{2}}=E^{2} . G^{\mu \nu}$ is the metric which appears in the noncommutative action derived from the background solution. In the background solution, we take a gauge where the solution is independent of the time and the D-string is along $\sigma=x^{1}$ coordinate. Here we do not have to take the Seiberg-Witten limit, but the same metric follows from the limit as mentioned before.

Now let us study the behavior of the open string coupling, $G_{s}$, in the decoupling limit. The open string coupling in terms of the closed string coupling, $g_{s}$ is given by [21]

$$
\begin{aligned}
G_{s} & =g_{s} \sqrt{\frac{\operatorname{det}(G+\lambda \Phi)}{\operatorname{det}(g+\lambda B)}} \\
& =g_{s} \sqrt{\frac{\operatorname{det}(g+\lambda B)}{\operatorname{det} g}} .
\end{aligned}
$$


Note that the second line of the above equation is true for any choice of $\Phi$. Then, imposing the criticality condition (6.1)

$$
\operatorname{det}(g+\lambda B)=-\left|g_{t}\right| g_{x}(\lambda B)^{2}, \quad \operatorname{det} g=-\left|g_{t}\right| g_{x} g_{y} g_{z},
$$

and hence,

$$
G_{s}=g_{s} \sqrt{\frac{(\lambda B)^{2}}{g_{y} g_{z}}} .
$$

In the Seiberg-Witten decoupling limit (6.4), $G_{s} \sim g_{s} \epsilon^{-1 / 2}$. Therefore, in order to have a finite open string coupling, the closed string coupling $g_{s}$ should behave as

$$
g_{s} \sim \sqrt{\epsilon} .
$$

In other words in the decoupling limit of (6.4) and (6.12) the closed strings are essentially decoupled while the open strings, which are of finite coupling, govern the dynamics of the system.

We would like to comment that unlike the usual belief that for $\mathbf{E} \cdot \mathbf{B} \neq 0$ the Seiberg-Witten decoupling limit makes the Born-Infeld action imaginary and unstable, is not necessarily true. The argument of Ref. [22] does not work for the case here because it is based on the uniform scaling of $g_{\mu \nu}$. The above computation explicitly demonstrates that there may be a decoupling limit of noncommutative worldvolume gauge theories even for $\mathbf{E} \cdot \mathbf{B} \neq 0$.

\section{NCOS limit}

It is known that strings living in an electric field background in a particular limit decouples from the closed strings [23]. In this limit the electric field is taken to some "critical limit" - and consequently the closed strings mass scale goes to infinity, while open strings remain of finite mass. In this limit the closed string coupling, $g_{s}$ also goes to infinity [23]. As we will show, although in our case the electric field is already fixed by the supersymmetry and BPS conditions, still our D3-D3 system admits an NCOS limit.

To show that such a limit where the closed strings decouple exists, let us consider the following limit:

$$
\begin{aligned}
& g_{t} \sim g_{z} \sim \lambda E \sim \lambda^{a} \\
& g_{x} \sim g_{y} \sim \lambda, \quad B, \quad \tilde{B}=\text { fixed }, \lambda=2 \pi \alpha^{\prime} \rightarrow 0 .
\end{aligned}
$$

with $a<1$. The closed string degrees decouple because their mass scale

$$
g_{t} / \alpha^{\prime} \sim \lambda^{a-1}
$$

goes to infinity as $\lambda \rightarrow 0$.

\footnotetext{
${ }^{6}$ Note that this criticality in principle is different from ours, Eq.(6.1).
} 
In this scaling limit, the open string metric can be read from Eq. (6.3): 凤

$$
G^{\mu \nu}=\frac{1}{g_{x}}\left(\begin{array}{cccc}
-u v & 0 & w \sqrt{u} & 0 \\
0 & 1 & 0 & \tilde{B} / B \\
w \sqrt{u} & 0 & 0 & 0 \\
0 & \tilde{B} / B & 0 & v
\end{array}\right)
$$

where we have defined

$$
u \equiv g_{z} /\left|g_{t}\right|, \quad v \equiv K /(\lambda B)^{2}, \quad w \equiv g_{x} /(\lambda B)
$$

It is clear that $\alpha^{\prime} G_{t}^{-1}$ remains finite in the scaling limit. Hence all the massive modes of open strings are of finite mass and hence limit (7.1) may lead to an NCOS theory. We would like to note that although one of the diagonal elements of the metric (7.3) is zero,

$$
\operatorname{det} G_{\mu \nu}=-\frac{g_{x}\left|g_{t}\right|}{g_{y} g_{z}}(\lambda B)^{4}
$$

and hence metric is not singular. The noncommutativity parameter

$$
\theta^{\mu \nu}=\frac{\lambda}{g_{x}}\left(\begin{array}{cccc}
0 & \sqrt{u} \tilde{B} / B & 0 & v \sqrt{u} \\
-\sqrt{u} \tilde{B} / B & 0 & 0 & 0 \\
0 & 0 & 0 & -w \\
-v \sqrt{u} & 0 & w & 0
\end{array}\right)
$$

remains finite and also includes the spacetime component. As we see

$$
\lambda_{e f f}=\frac{\lambda}{g_{x}}
$$

is the open string mass scale, as well as the noncommutativity scale. Without loss of generality, one may consistently set $u=v=1$.

To complete our arguments, we still need to show that in the NCOS limit (7.1) the open string coupling is finite and also the massless closed string states have vanishing coupling. The open string coupling (6.11) in the above NCOS limit behaves as

$$
G_{s} \sim g_{s} \lambda^{\frac{1-a}{2}} .
$$

Therefore to have a finite $G_{s}$ the closed string coupling should behave as

$$
g_{s} \sim \lambda^{-\frac{1-a}{2}} \rightarrow \infty
$$

Similarly to the usual NCOS cases [23], our NCOS theory is actually defined in the strongly coupled closed strings regime. However, one should note that the coupling for the massless closed string (supergravity) modes, the ten-dimensional Newton constant $G_{N}$, differs from $g^{2}$ in factors coming

\footnotetext{
${ }^{7}$ In this section we set $\Phi=0$, and hence $G^{\mu \nu}$ and $\theta^{\mu \nu}$ are just the symmetric and anti-symmetric parts of $(g+\lambda B)^{-1}$ respectively.
} 
from $\sqrt{\operatorname{det} g_{10}}$. In order to work out the effective coupling for this massless modes, we first need to fix the scaling of the components of the closed string metric transverse to the D3-D3 system. Let

$$
g_{a b}=g_{\perp} \delta_{a b}, \quad a, b=1,2 \cdots, 6 .
$$

Then the scaling behavior of $g_{\perp}$ can be fixed demanding that the D3-brane is still carrying one unit of RR-charge. This implies that

$$
g_{\perp} \sim \lambda^{\frac{1+a}{2}}
$$

On the other hand the effective coupling for the gravitons polarized, say in $t$ - $z$ directions would be

$$
\begin{aligned}
g_{t} G_{N} & =\frac{g_{s}^{2} \lambda^{4}}{g_{\perp}^{3}} \sqrt{\frac{g_{t}}{g_{x} g_{y} g_{z}}} \\
& \sim \lambda^{\frac{1-a}{2}} \rightarrow 0 .
\end{aligned}
$$

That is, in the decoupling limit defined through (7.1), (7.8), the massless closed strings will also decouple. Therefore, all the closed strings, massless and massive, are decoupled and our theory is completely described by the open strings with mass scale $\lambda_{\text {eff }}$ and the coupling $G_{s}$ given in Eq. (6.11).

The above analysis demonstrates that there exists an NCOS limit related to the supersymmetric D3- $\overline{\mathrm{D} 3}$. Note that, in the above computation, $\tilde{B}$ does not play an essential role and may be set to zero. This in particular implies that upon a T-duality in the $x^{1}$ direction our NCOS theory is mapped into another NCOS theory coming from the supersymmetric D2- $\overline{\mathrm{D} 2}$ system of Ref. [4, 6].

\section{Discussion}

In this paper we have studied various generalizations of the supertubes and brane-antibrane systems. We have shown that there is a $1 / 4$ BPS D3-D3 configuration in which $\mathbf{E} \cdot \mathbf{B} \neq 0$, where $\mathbf{E}$ and $\mathbf{B}$ are the electric and magnetic background fields and therefore if we choose $\mathbf{E}$ to be along the $z$ direction, the system is specified with two components of the $\mathbf{B}$ field, one parallel to $\mathbf{E}$ and the other one transverse to $\mathbf{E}$. The latter may be chosen to be in the $x$ direction. Analyzing the system through the Born-Infeld action and also the matrix model we showed that in general the $B$-field component parallel to $\mathbf{E}$ can be an arbitrary function of $x$ and $y$ while the other component can be an arbitrary function of $y$. Working out the spectrum of the open strings attached to D3 and D3 branes we showed that in the $E \rightarrow 1$ limit tachyon disappears from the spectrum, which is in agreement with our earlier discussions. Here we mainly focused on the flat D3- $\overline{\mathrm{D} 3}$ system. However similarly to the supertubes case one may think of generalizations with other cross sectional shapes. Although we have discussed the D3- $\overline{\mathrm{D} 3}$ case explicitly, it is also straightforward to generalize our discussions to the higher-dimensional brane-antibrane systems, the simplest of which can be obtained through T-dualities.

\footnotetext{
${ }^{8}$ It is straightforward to verify that the effective coupling for the other graviton polarizations as well as the other supergravity modes will go to zero as some power of $\lambda^{\frac{1-a}{2}}$.
} 
The supergravity solution for the supertubes has also been worked out [24]. The similar problem can be studied for the general D3- $\overline{\mathrm{D} 3}$ system we have introduced here.

Here we also briefly discussed the systems with lower supersymmetries. In particular, we studied the 1/8 BPS solutions in the context of matrix model. These solutions are matrix version of the noncommutative tube-D4 system studied in [12]. Another possible generalization is on supertubes or D2- $\overline{\mathrm{D} 2}$ connecting many D4 branes separated in the transverse space [25], which appear as electrically charged magnetic strings from the view point of the D4 worldvolume. In the same lines, generalizing the arguments of section 4, one may construct 6 or 8 dimensional BPS solutions 26] corresponding to D0-D6 or D0-D8 systems. Such solutions can be 1/16, 1/8 or 3/16 BPS. However, the general classification of the BPS equations involving electric components is a complicated issue and may be studied in an independent work.

As another interesting direction to pursue, one may study the behavior of the D3- $\overline{\mathrm{D} 3}$ system under S-duality of type IIB strings. Being BPS and since S-duality generally does not change the number of conserved supercharges, we expect under S-duality D3- $\overline{\mathrm{D} 3}$ system to be mapped into another stable 1/4 BPS configuration. In fact using the results of Ref. [27], one can show that under the S-duality transformation $g_{s} \rightarrow \frac{1}{g_{s}}$, the BPS condition $E=1$, is again mapped into another similar BPS condition. To be precise, the background $B$-field of (5.1) after the S-duality becomes

$$
B_{01}=\operatorname{sign}(B), \quad B_{12}=\frac{1+\tilde{B}^{2}}{|B|}, \quad B_{23}=\tilde{B} \operatorname{sign}(B)
$$

and all the other components zero. As we see again the electric field, which is now in $x^{1}$ direction, squares to one.? It is then, straightforward to show that for a general $S L(2, Z)$ S-duality transformation we have similar results; however, we may have non-zero $B_{23}$ or $B_{13}$ fields as well. Therefore, the set of general $\mathbf{E} \cdot \mathbf{B} \neq 0$ supersymmetric D3- $\overline{\mathrm{D} 3}$ systems is invariant under $S L(2, Z)$ S-duality. Then, one may take the NCSYM or NCOS limits. We expect that generically these $1 / 4$ BPS NCOS theories also enjoy the $S L(2, Z)$ symmetry, just similar to the usual $1 / 2$ BPS NCOS theories [28, 29]. However, for some particular values of the background fields, we expect the NCOS theory to be S-dual to an NCSYM [29, 30]. The $S L(2, Z)$ behavior of the BPS D3-D3 system deserves a more detailed study which we postpone to future works.

\section{Acknowledgments:}

We would like to thank K. Lee, T. Yanagida and P. Yi for enlightening discussions. The work of D.B. was supported in part by the Korea Research Foundation Grant KRF-2002-013-D00028. That of N.O. was supported in part by a Grant-in-Aid for Scientific Research No. 12640270. M.M.ShJ. was supported in part by NSF grant PHYS-9870115 and in part by funds from the Stanford Institute for Theoretical Physics.

\section{References}

\footnotetext{
${ }^{9}$ We would like to note that the above expressions are for the S-dual of the $B$ field on the brane, for the antibrane they are $B_{01}=\operatorname{sign}(B), B_{12}=-\frac{1+\tilde{B}^{2}}{|B|}$ and $B_{23}=\tilde{B} \operatorname{sign}(B)$.
} 
[1] J. Polchinski, "Dirichlet-Branes and Ramond-Ramond Charges," Phys. Rev. Lett. 75 (1995) 4724, arXiv:hep-th/9510017.

[2] D. Mateos and P. K. Townsend, "Supertubes," Phys. Rev. Lett. 87, 011602 (2001) arXiv:hep$\operatorname{th} / 0103030$.

[3] D. Bak and K. Lee, "Noncommutative Supersymmetric Tubes," Phys. Lett. B 509, 168 (2001) arXiv:hep-th/0103148.

[4] D. Bak and A. Karch, "Supersymmetric Brane-Antibrane Configurations," Nucl. Phys. B626, 165 (2002) arXiv:hep-th/0110039.

[5] D. Mateos, S. Ng and P. K. Townsend, "Tachyons, Supertubes and Brane/Antibrane Systems," JHEP 0203, 016 (2002) arXiv:hep-th/0112054.

[6] D. Bak and N. Ohta, "Supersymmetric D2 Anti-D2 Strings," Phys. Lett. B527, 131 (2002) arXiv:hep-th/0112034.

[7] D. Bak and S. W. Kim, "Junctions of Supersymmetric Tubes," Nucl. Phys. B622, 95 (2002) arXiv:hep-th/0108207.

[8] J.H. Cho and P. Oh, "Super D-helix," Phys. Rev. D 64, 106010 (2001) arXiv:hep-th/0105095.

[9] I. Bena, "The Polarization of F1 Strings into D2-branes: 'Aut Caesar aut nihil', "arXiv:hep$\operatorname{th} / 0111156$.

[10] D. Mateos, S. Ng and P. K. Townsend, "Supercurves," arXiv:hep-th/0204062.

[11] Y. Hyakutake and N. Ohta, "Supertubes and Supercurves from M-Ribbons," Phys. Lett. B539 (2002) 153, arXiv:hep-th/0204161.

[12] M. Kruczenski, R. C. Myers, A. W. Peet and D. J. Winters, "Aspects of Supertubes," JHEP 0205, 017 (2002) arXiv:hep-th/0204103.

[13] R. Dijkgraaf, E. Verlinde, H. Verlinde, "Matrix String Theory," Nucl. Phys. B500 (1997) 43, arXiv:hep-th/9703030.

[14] N. Seiberg and E. Witten, "String Theory and Noncommutative Geometry," JHEP 9909, 032 (1999) arXiv:hep-th/9908142.

[15] T. Banks, W. Fischler, S. H. Shenker and L. Susskind, "M-Theory as a Matrix Model: A Conjecture," Phys. Rev. D55, 5112 (1997) arXiv:hep-th/9610043.

[16] N. Seiberg, "Why is the Matrix Model Correct?," Phys. Rev. Lett. 79, 3577 (1997) arXiv:hepth/9710009.

[17] D. J. Gross and N. A. Nekrasov, "Dynamics of Strings in Noncommutative Gauge Theory," JHEP 0010, 021 (2000) arXiv:hep-th/0007204.

[18] L. Susskind, "The Quantum Hall Fluid and Noncommutative Chern-Simons Theory," arXiv:hep-th/0101029. 
[19] E. Bergshoeff and P. K. Townsend, "Super D-branes," Nucl. Phys. B490, 145 (1997) arXiv:hep-th/9611173.

[20] N. D. Lambert and D. Tong, "Dyonic Instantons in Five-dimensional Gauge Theories," Phys. Lett. B 462, 89 (1999) arXiv:hep-th/9907014; E. Eyras, P. K. Townsend and M. Zamaklar, "The Heterotic Dyonic Instanton," JHEP 0105, 046 (2001) arXiv:hep-th/0012016.

[21] N. Seiberg, "A Note on Background Independence in Noncommutative Gauge Theories, Matrix Model and Tachyon Condensation," JHEP 0009, 003 (2000) arXiv:hep-th/0008013.

[22] O. Aharony, J. Gomis and T. Mehen, "On Theories with Light-Like Noncommutativity," JHEP 0009, 023 (2000) arXiv:hep-th/0006236.

[23] N. Seiberg, L. Susskind, N. Toumbas, "Strings in Background Electric Field, Space/Time Noncommutativity and a New Noncritical String Theory," JHEP 0006, 021 (2000) arXiv:hepth/0005040.

R. Gopakumar, J. Maldacena, S. Minwalla, A. Strominger, "S-Duality and Noncommutative Gauge Theory," JHEP 0006, 036 (2000) arXiv:hep-th/0005048.

[24] R. Emparan, D. Mateos and P.K. Townsend, "Supergravity Supertubes," JHEP 0107, 011 (2001) arXiv:hep-th/0106012.

[25] D. Bak and K. Lee, "Supertubes connecting D4 branes," arXiv:hep-th/0206185.

[26] D. Bak, K. Lee and J. H. Park, "BPS Euations in Six and Eight Dimensions," arXiv:hepth/0204221].

[27] M. B. Green and M. Gutperle, "Comments on Three-Branes," Phys.Lett. B377 (1996) 28, arXiv:hep-th/9602077.

[28] J. G. Russo and M. M. Sheikh-Jabbari, "On Noncommutative Open String Theories," JHEP 0007 (2000) 052, arXiv:hep-th/0006202.

[29] R.-G. Cai and N. Ohta, "(F1, D1, D3) Bound States, Its Scaling Limits and $S L(2, \mathbf{Z})$ Duality", Prog. Theor. Phys. 104 (2000) 1073, arXiv:hep-th/0007106.

[30] J. X. Lu, S. Roy and H. Singh, " $S L(2, \mathbf{Z})$ duality and 4-dimensional noncommutative theories", Nucl. Phys. B595 (2001) 298, arXiv:hep-th/0007168. 